\title{
Observation of coherent interfacial optical phonons in GalnP/GaAs/GalnP single quantum wells
}

\author{
Y.-M. Chang ${ }^{\text {a) }}$ \\ Center for Condensed Matter Sciences, National Taiwan University, Taipei 10673, Taiwan, \\ Republic of China \\ H. H. Lin \\ Department of Electrical Engineering, National Taiwan University, Taipei 10673, Taiwan, Republic of China \\ C. T. Chia \\ Department of Physics, National Taiwan Normal University, Taipei 116, Taiwan, Republic of China \\ Y. F. Chen \\ Department of Physics, National Taiwan University, Taipei 10673, Taiwan, Republic of China
}

(Received 14 October 2003; accepted 18 February 2004)

\begin{abstract}
Coherent phonon spectroscopy of $\mathrm{GaInP} / \mathrm{GaAs} / \mathrm{GaInP}$ single quantum wells is demonstrated with time-resolved second-harmonic generation. Coherent longitudinal optical phonons are impulsively launched via transient pump-induced field screening and stimulated Raman scattering in the well and barrier regions. A phonon mode at $9.4 \mathrm{THz}$ is identified as interfacial phonon localized in the GaAs/GaInP hetero-interface. The free induced dephasing of this coherent interfacial phonon is analyzed with window-gated Fourier transform. Its dephasing time is used to characterize the interface quality of semiconductor heterostructures. (C) 2004 American Institute of Physics.
\end{abstract}

[DOI: $10.1063 / 1.1704859$ ]

The heterostructures comprised of GaAs and latticematched $\mathrm{Ga}_{x} \operatorname{In}_{1-x} \mathrm{P}(x \sim 0.5)$ have attracted a lot of attention because of the ordering induced band alignment. ${ }^{1-8}$ The ordering of GaInP depends on the growth parameters such as growth temperature, III/V ratio, growth rate, and substrate orientation. $\mathrm{Ga}_{x} \mathrm{In}_{1-x} \mathrm{P}(x \sim 0.5)$ can form a spontaneously partially ordered alloy when grown on (001) oriented GaAs substrate under suitable growth condition. ${ }^{2}$ The band gap of the ordered GaInP is smaller by as much as $0.1 \mathrm{eV}$ than that of the disordered GaInP alloy. The band alignment changes from type I for the disordered $\mathrm{GaInP} / \mathrm{GaAs}$ heterostructure to type II for the ordered GaInP/GaAs heterostructure. ${ }^{3}$ However, it is difficult to grow sharp GaInP/GaAs interfaces because of the In memory effect, the P-to-As exchange process, and the diffusion of As atoms into the bulk GaInP, during the interface formation. ${ }^{4}$ A low-band-gap ternary or quaternary interlayer forms spontaneously during the sample growth. It is a general reason for the deterioration of the $\mathrm{GaInP} / \mathrm{GaAs}$ interface quality.

The optical property of $\mathrm{GaInP} / \mathrm{GaAs} / \mathrm{GaInP}$ single quantum wells (SQWs) has been studied in many aspects of continuous-wave laser spectroscopy, such as photoluminescence, ${ }^{5-7}$ photoreflectance, ${ }^{8}$ and spontaneous Raman scattering, ${ }^{2}$ but not in the femtosecond laser spectroscopy. The development of femtosecond laser system has made it possible to generate and detect coherent phonons in semiconductor nanostructures with time-resolved pump and probe technique. ${ }^{9}$ The phonon frequency and dephasing time can be directly determined from the temporal domain data. The transient lattice vibration and its interaction with the structure defects, impurities, and photoexcited carriers can be in-

${ }^{a)}$ Electronic mail: ymchang@ccms.ntu.edu.tw vestigated in real time. In this letter, we demonstrate the coherent phonon spectroscopy of GaInP/GaAs/GaInP SQWs with time-resolved second-harmonic generation (TRSHG) technique. This technique has been used to study the carrier and phonon dynamics at clean surfaces or buried interfaces because of its intrinsic surface and interface sensitivity. ${ }^{10}$

The studied sample structure from the top to the bottom is $0.2 \mu \mathrm{m} \mathrm{Ga}_{0.48} \mathrm{In}_{0.52} \mathrm{P}, 7 \mathrm{~nm} \mathrm{GaAs}, 1 \mu \mathrm{m} \mathrm{Ga}_{0.48} \mathrm{In}_{0.52} \mathrm{P}, 0.5$ $\mu \mathrm{m}$ GaAs buffer, and SI GaAs (100) substrate. The sample growth temperature is $520^{\circ} \mathrm{C}$. The photoluminescence (PL) spectrum taken at $15 \mathrm{~K}$ indicates two major PL peaks at 1.52 and $1.96 \mathrm{eV}$, which correspond to the band-edge transitions in the GaAs well and GaInP barrier, respectively. ${ }^{4}$ No deep emission band below GaAs band gap is observed. ${ }^{6}$ This PL result suggests that the studied SQW sample has disordered GaInP structure and the band alignment is type I. A modelocked Ti-sapphire laser system, delivering $800 \mathrm{~nm}(h \nu$ $=1.56 \mathrm{eV}$ ), $82 \mathrm{MHz}, 30 \mathrm{fs}$ pulse train, is used as the light source for the pump and probe experiments. In the experiments, the pump and probe pulses are set to $s$-polarized and $p$-polarized, respectively. They are set to parallel to each other and incident to the sample at $45^{\circ}$ angle. The pump beam is intensity modulated with a mechanical chopper. The reflected SH signal in the probe is detected with a thermal electric cooled photomultiplier tube (PMT) in $p_{\text {in }}-p_{\text {out }}$ geometry. The pump-induced SH signal is obtained by processing the PMT output with a lock-in amplifier and recorded as a function of the delay time between the pump and probe.

Figure 1(a) shows a typical TRSHG measurement and its analysis. The oscillatory component, $S_{\text {osc }}(t)$, extracted from the slowly varying background, is shown in the inset. Its Fourier power spectrum [curve A in Fig. 1(b)] reveals six 

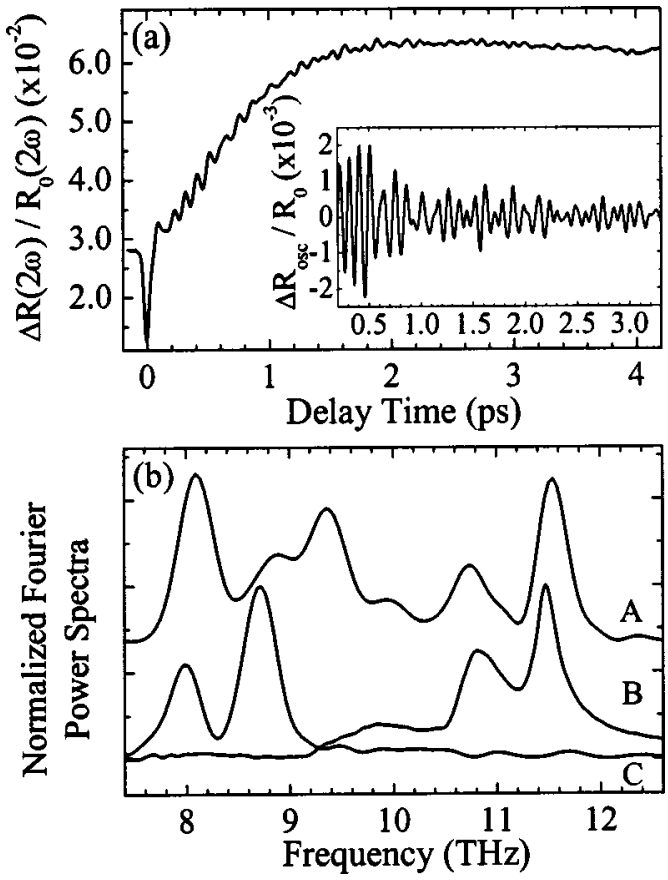

FIG. 1. (a) A typical TRSHG measurement on a GaInP/GaAs/GaInP SQW. Its oscillatory component is shown in the inset. (b) Normalized phonon spectra of TRSHG measurement (curve A/with offset), spontaneous Raman scattering (curve B), and time-resolved reflectivity change measurement (curve C).

coherent optical phonon modes created by the intensive pump pulse. These six phonon modes are tentatively assigned to GaP-like LO phonon (11.5 THz), InP-like LO phonon $(10.7 \mathrm{THz})$, and LO-plasmon coupling mode (LOPC, $10.0 \mathrm{THz}$ ) in the InGaP barrier, interfacial phonon (IP, 9.4 $\mathrm{THz})$ in the GaAs/GaInP hetero-interface, LO phonon $(8.8$ $\mathrm{THz}$ ) in the GaAs well, and LO-plasmon coupling mode (8.0 $\mathrm{THz}$ ) in the GaAs buffer layer. The results of polarizationdependent and power-dependent TRSHG measurements indicate that impulsive stimulated Raman scattering and transient depletion field screening are both responsible for launching these phonon modes. ${ }^{11}$

Spontaneous Raman scattering and time-resolved reflectivity change measurements are performed on the same sample to verify our phonon assignment. Spontaneous Raman spectroscopy [curve B in Fig. 1(b)] is carried out at room temperature using an argon-ion laser emitting at 514 $\mathrm{nm}$. The (100) backscattering Raman spectrum reveals three Raman-active bulk phonon modes, which are GaP-like LO phonon at $11.5 \mathrm{THz}$, InP-like LO phonon at $10.7 \mathrm{THz}$, and a broadened TO phonon band at $10.0 \mathrm{THz}$, in the GaInP barrier. $^{2}$ On the other hand, time-resolved reflectivity change measurement is performed under the same experimental configuration of TRSHG. The measured reflectivity change $(\Delta R)$ in the probe is mainly governed by the pump-induced absorption and dielectric response in the GaAs regions. Its coherent phonon spectrum [curve $\mathrm{C}$ in Fig. 1(b)] reveals two phonon modes: bulk LO phonon $(8.8 \mathrm{THz})$ in the GaAs well and LO-plasmon coupling mode $(8.0 \mathrm{THz})$ in the GaAs buffer layer. ${ }^{12}$ These two experiments confirm most of our phonon assignment.

The phonon peak at $9.4 \mathrm{THz}$ cannot be assigned to any well-known bulk phonon mode in the SQW. Its frequency

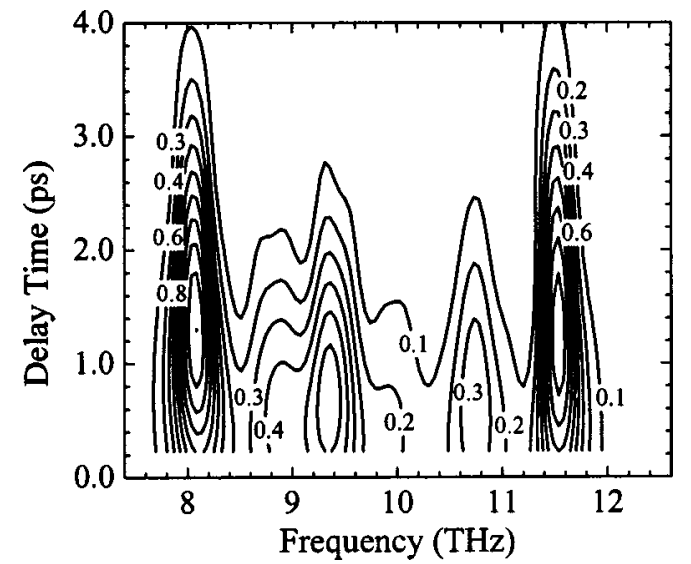

FIG. 2. Contour plot of the window-gated Fourier transform $S\left(f, \tau_{d}\right)$ of the oscillatory component shown in Fig. 1(a) inset. The contour plot is normalized to the maximum peak intensity of GaAs LOPC mode at $8.0 \mathrm{THz}$.

always remains the same when the pump-injected carrier density varies from $5 \times 10^{17}$ to $2 \times 10^{19} / \mathrm{cm}^{3}$, which is estimated in the GaAs region. This observation excludes any assignment to any possible phonon-plasmon coupling mode in the SQW. ${ }^{13}$ In fact, this phonon peak can be identified as a minute shoulder in the spontaneous Raman spectrum. We therefore assign this phonon peak to an interfacial optical phonon mode localized in the GaAs/GaInP hetero-interface. It is confined in the ternary or quaternary interlayer, which is formed during the sample growth. ${ }^{4}$ This interfacial phonon shall be Raman-active. The comparison of peak intensities in Fig. 1(b) phonon spectra, however, suggests that the transient depletion field screening is the dominant driving mechanism to launch this coherent interfacial phonon in our TRSHG experiments.

We develop a fitting method to eliminate the ambiguity in determining the oscillatory amplitude and dephasing time of coherent phonons when two or more phonon modes coexist in the oscillatory component, $S_{\text {osc }}(t)$. The idea is to perform the fitting in the spectral domain instead of the tempo-
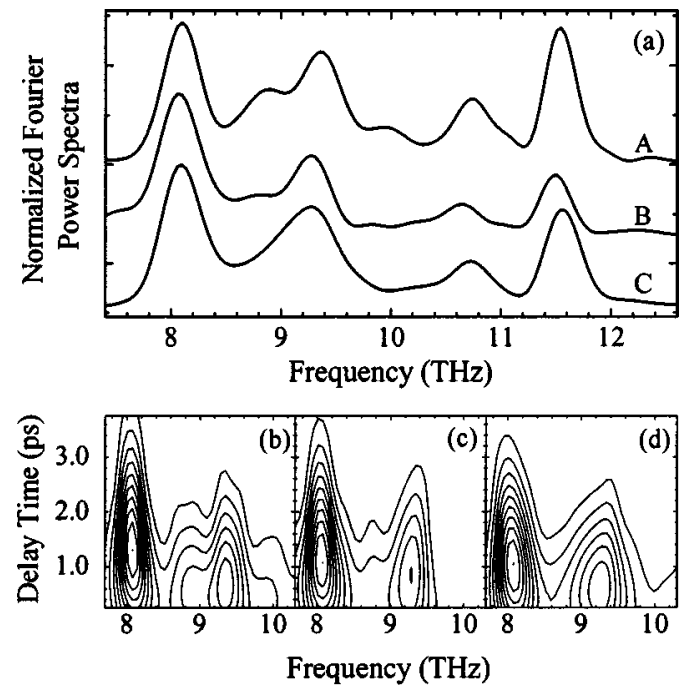

FIG. 3. (a) Fourier power spectra of three GaInP/GaAs/GaInP SQWs: sample A $\left(520^{\circ} \mathrm{C}\right)$, sample B $\left(480^{\circ} \mathrm{C}\right)$, and sample $\mathrm{C}\left(440^{\circ} \mathrm{C}\right)$, respectively. The spectra (with offset) are all normalized to their maximum peak intensity of GaAs LOPC mode at $8.0 \mathrm{THz}$. Their window-gated Fourier transforms are plotted in (b) sample A, (c) sample B, and (d) sample C, respectively. 
TABLE I. List of the dephasing times of coherent phonons of three GaInP/GaAs/GaInP SQWs, which have different sample growth temperatures.

\begin{tabular}{ccccccc}
\hline \hline Sample & $\begin{array}{c}\text { Growth } \\
\text { temperature } \\
\left({ }^{\circ} \mathrm{C}\right)\end{array}$ & $\begin{array}{c}\mathrm{LOPC}_{\mathrm{GaAs}} \\
(8.0 \mathrm{THz}) \\
(\mathrm{ps})\end{array}$ & $\begin{array}{c}\mathrm{LO}_{\mathrm{GaAs}} \\
(8.8 \mathrm{THz}) \\
(\mathrm{ps})\end{array}$ & $\begin{array}{c}\mathrm{IP} \text { mode } \\
(9.4 \mathrm{THz}) \\
(\mathrm{ps})\end{array}$ & $\begin{array}{c}\mathrm{LO}_{\text {InP-like }} \\
(10.7 \mathrm{THz}) \\
(\mathrm{ps})\end{array}$ & $\begin{array}{c}\mathrm{LO}_{\mathrm{GaP}-\mathrm{like}} \\
(11.5 \mathrm{THz}) \\
(\mathrm{ps})\end{array}$ \\
\hline $\mathrm{A}$ & 520 & $1.6 \pm 0.2$ & $0.6 \pm 0.2$ & $0.8 \pm 0.2$ & $0.8 \pm 0.2$ & $1.7 \pm 0.2$ \\
$\mathrm{~B}$ & 480 & $1.4 \pm 0.2$ & $0.5 \pm 0.2$ & $0.9 \pm 0.2$ & $0.8 \pm 0.2$ & $1.8 \pm 0.2$ \\
$\mathrm{C}$ & 440 & $1.0 \pm 0.2$ & $\cdots$ & $0.6 \pm 0.2$ & $0.7 \pm 0.2$ & $1.3 \pm 0.2$ \\
\hline \hline
\end{tabular}

ral domain. The oscillatory component, $S_{\text {osc }}(t)$, is converted into the spectral domain with window-gated Fourier transform technique: ${ }^{14}$

$$
\begin{aligned}
& S\left(f, \tau_{d}\right)=\left|\int_{\tau_{0}}^{\tau_{1}} S_{\mathrm{osc}}(t) G\left(\sigma, t, \tau_{d}\right) e^{i 2 \pi f t} d t\right|, \\
& \tau_{d} \in\left[\tau_{0}, \tau_{1}\right],
\end{aligned}
$$

where $G\left(\sigma, t, \tau_{d}\right)$ denotes the scanning Gaussian window: $(\sqrt{2 \pi} \sigma)^{-1} \exp \left[-\left(t-t_{d}\right)^{2} /\left(2 \sigma^{2}\right)\right], \tau_{d}$ and $\sigma$ define the scanning peak position and rms width of the gating window, and $\left[\tau_{0}, \tau_{1}\right]$ is the temporal bounds of $S_{\text {osc }}(t)$. The time evolution of each coherent phonon can now be visualized with this analysis technique. For example, Fig. 2 shows the contour plot of $S\left(f, \tau_{d}\right)$ performed on the oscillatory data shown in the inset of Fig. 1(a). The window parameters are set to $\tau_{0}=0.237 \mathrm{ps}, \tau_{1}=5.28 \mathrm{ps}$, and $\sigma=1.274 \mathrm{ps}$. Least-squares fitting routine is then performed in the spectral domain data, $S\left(f_{\mathrm{ph}}, \tau_{d}\right)$, for a selected coherent phonon mode, $f_{\mathrm{ph}}$. The fitting function $F\left(f_{\mathrm{ph}}, \tau_{d}\right)$ is defined as

$$
\begin{aligned}
F\left(f_{\mathrm{ph}}, \tau_{d}\right)= & \mid \int_{\tau_{0}}^{\tau_{1}} A e^{-t / T} \cos \left(2 \pi f_{\mathrm{ph}} t+\varphi\right) \\
& \times G\left(\sigma, t, \tau_{d}\right) e^{i 2 \pi f_{\mathrm{ph}} t} d t \mid, \quad \tau_{d} \in\left[\tau_{0}, \tau_{1}\right],
\end{aligned}
$$

where $A, T$, and $\varphi$ are the fitting parameters: oscillatory amplitude, dephasing time, and initial phase of the selected coherent phonon mode $f_{\text {ph }}$, respectively.

Furthermore, we study three SQW samples, which have identical layer structure but are grown at three different temperatures. Figure 3 plots their coherent phonon spectra and window-gated Fourier transforms. The contour plots clearly illustrate the oscillatory strength of GaAs LO phonon (8.0 $\mathrm{THz})$ and GaAs/GaInP interfacial phonon (9.4 THz). Table I lists the dephasing times obtained with the aforementioned fitting method. The fitting results clearly reveal the general trend that the higher the sample growth temperature, the longer the phonon dephasing times.

In general, the dephasing of coherent phonon can be through the following processes: phonon-phonon scattering, ${ }^{15}$ phonon-carrier scattering, ${ }^{13}$ and phonon-impurity scattering. ${ }^{16}$ Our study concludes that the oscillatory strength of this interfacial phonon is determined by the atomic structure in the GaAs/GaInP hetero-interface. The deterioration of $\mathrm{GaAs} / \mathrm{InGaP}$ interface quality is due to the formation of lowband-gap ternary (InGaAs) or quaternary (InGaAsP) interlayer between the InGaP barrier and GaAs well. ${ }^{4}$ The As-to-P exchange process makes it difficult to grow sharp GaInP/ GaAs interface. The imperfection of this interlayer leads to the rapid dephasing of the coherent interfacial phonon.

In summary, we investigate the phonon dynamics of GaInP/GaAs/GaInP SQWs with TRSHG. A localized phonon mode is identified in the GaInP/GaAs hetero-interface. The dephasing time of coherent phonons shows strong correlation to the sample growth temperature. The atomic structure and interface quality can significantly influence the observed coherent phonon spectroscopy. This report demonstrates the capability of TRSHG to study the phonon dynamics in the buried interfaces of semiconductor nanostructures.

The authors acknowledge the financial support of National Science Council, Academia Sinica, and Ministry of Education, of Taiwan, R.O.C.

${ }^{1}$ Y. Zhang, A. Mascarenhas, and L. W. Wang, Appl. Phys. Lett. 80, 3111 (2002).

${ }^{2}$ G. R. Moriarty, M. Kildemo, J. T. Beechinor, M. Murtagh, P. V. Kelly, G. M. Crean, and S. W. Bland, Thin Solid Films 364, 244 (2000).

${ }^{3}$ J. Zeman, G. Martinez, P. Y. Yu, S. H. Kwok, and K. Uchida, Phys. Status Solidi B 211, 239 (1999).

${ }^{4}$ R. Kúdela, M. Kučera, B. Olejníková, P. Eliáš, S. Hasenöhrl, and J. Novák, J. Cryst. Growth 212, 21 (2000).

${ }^{5}$ J. Chen, J. R. Sites, I. L. Spain, M. J. Hafich, and G. Y. Robinson, Appl. Phys. Lett. 58, 744 (1991).

${ }^{6}$ H. M. Cheong and K. Matsumoto, Phys. Rev. B 62, 1536 (2000).

${ }^{7}$ Q. Liu, S. Derksen, A. Lindner, F. Scheffer, W. Prost, and F. J. Tegude, J. Appl. Phys. 77, 1154 (1995).

${ }^{8}$ G. Arnaud, P. Boring, B. Gil, J. Carcia, J. Landesman, and M. Leroux, Phys. Rev. B 46, 1886 (1992).

${ }^{9}$ T. Dekorsy, G. C. Cho, and H. Hurz, in Light Scattering in Solids VIII (Springer, Berlin, 2000), and references therein.

${ }^{10}$ Y.-M. Chang, L. Xu, and H. W. K. Tom, Phys. Rev. B 59, 12220 (1999).

${ }^{11}$ K. J. Yee, Y. S. Lim, T. Dekorsy, and D. S. Kim, Phys. Rev. Lett. 86, 1630 (2001).

${ }^{12}$ T. Dekorsy, A. M. T. Kim, G. C. Cho, H. Kurz, A. V. Kuznetsov, and A. Förster, Phys. Rev. B 53, 1531 (1996).

${ }^{13}$ Y.-M. Chang, Appl. Phys. Lett. 80, 2487 (2002).

${ }^{14}$ M. Hase, M. Kitajima, S. Nakashima, and K. Mizoguchi, Phys. Rev. Lett. 88, 067401 (2002).

${ }^{15}$ Y.-M. Chang, Appl. Phys. Lett. 82, 1781 (2003).

${ }^{16}$ M. Hase, K. Ishioka, and M. Kitajima, Appl. Phys. Lett. 76, 1258 (2000). 
Applied Physics Letters is copyrighted by the American Institute of Physics (AIP). Redistribution of journal material is subject to the AIP online journal license and/or AIP copyright. For more information, see http:/ojps.aip.org/aplo/aplcr.jsp

Copyright of Applied Physics Letters is the property of American Institute of Physics and its content may not be copied or emailed to multiple sites or posted to a listserv without the copyright holder's express written permission. However, users may print, download, or email articles for individual use. 